\title{
A High-Quality Mach-Zehnder Interferometer Fiber Sensor by Femtosecond Laser One-Step Processing
}

\section{Longjiang Zhao ${ }^{1}$, Lan Jiang ${ }^{1, *}$, Sumei Wang ${ }^{1}$, Hai Xiao ${ }^{2}$, Yongfeng Lu ${ }^{3}$ and Hai-Lung Tsai ${ }^{4}$}

1 School of Mechanical Engineering, Beijing Institute of Technology, 100081, China; E-Mails: zhaolongjiang@bit.edu.cn (L.Z.); wangsumei@bit.edu.cn (S.W.)

2 Department of Electrical and Computer Engineering, Missouri University of Science and Technology, Rolla, MO 65409, USA; E-Mail: xiaoha@mst.edu (H.X.)

3 Department of Electrical Engineering, University of Nebraska-Lincoln, Lincoln, NE 68588, USA; E-Mail: ylu2@unl.edu (Y.L.)

4 Department of Mechanical and Aerospace Engineering, Missouri University of Science and Technology, Rolla, MO 65409, USA; E-Mail: tsai@mst.edu (H.-L.T.)

* Author to whom correspondence should be addressed; E-Mail: jianglan@bit.edu.cn; Tel.: +86-010-68914517; Fax: +86-010-68914517.

Received: 14 October 2010; in revised form: 15 November 2010 / Accepted: 16 December 2010 / Published: 23 December 2010

\begin{abstract}
During new fiber sensor development experiments, an easy-to-fabricate simple sensing structure with a trench and partially ablated fiber core is fabricated by using an $800 \mathrm{~nm} 35 \mathrm{fs} 1 \mathrm{kHz}$ laser. It is demonstrated that the structure forms a Mach-Zehnder interferometer (MZI) with the interference between the laser light passing through the air in the trench cavity and that in the remained fiber core. The fringe visibilities are all more than $25 \mathrm{~dB}$. The transmission spectra vary with the femtosecond (fs) laser ablation scanning cycle. The free spectral range (FSR) decreases as the trench length increases. The MZI structure is of very high fabrication and sensing repeatability. The sensing mechanism is theoretically discussed, which is in agreement with experiments. The test sensitivity for acetone vapor is about $10^{4} \mathrm{~nm} / \mathrm{RIU}$, and the temperature sensitivity is $51.5 \mathrm{pm} /{ }^{\circ} \mathrm{C}$ at $200 \sim 875^{\circ} \mathrm{C}$ with a step of $25^{\circ} \mathrm{C}$.
\end{abstract}

Keywords: femtosecond laser; fiber sensors; Mach-Zehnder interferometer 


\section{Introduction}

Modified fiber substrates are widely used in sensors [1-4] and wavelength filters [5-7] through coupling with planar waveguides or resonators. With its advantages of small size, light weight, electromagnetic interference immunity, wide bandwidth, and low transmission loss an optical fiber is a preferred platform for micro-sensors. Furthermore, fiber optics are widely used to interface various optoelectronic components. There are three main ways of transforming a single-mode fiber into a desired component: (1) grinding by using abrasive powders [8]; (2) processing by using a femtosecond (fs) laser [9-11], and (3) etching with HF solution. Many transparent materials irradiated by fs laser pulses are ablated with property changes due to the strong nonlinear ionization and corresponding free electron generation [12,13]. Some Fabry-Perot interferometers have been successively fabricated in optical fibers with fs lasers and used for refractive index sensing [9,11,14]. The fiber optic localized plasmon resonance (FO-LPR) sensor is also reported in which D-shaped fibers engraved by a fs laser is adhered with Au nanoparticles [10,15]. A multi-d-shaped optical fiber for refractive index sensing in a communication grade multimode optical fiber is fabricated by using a fs laser [16].

In this study, a fs laser was used to fabricate fiber sensors, producing a MZI structure on fibers with exciting potentials in high-quality sensing of refractivity-sensitive parameters such as temperature, concentration, humidity, pressure, stress and strain. Characteristic transmission spectra demonstrate that the fringe visibilities are all more than $25 \mathrm{~dB}$. Fifty tested samples demonstrate the high fabrication and sensing repeatability of the simple sensing structure with some other advantages including reliability, compactness, robustness, high sensitivity, high flexibility, simple fabrication process and so on. The sensitivity for acetone vapor is about $10^{4} \mathrm{~nm} / \mathrm{RIU}$ (refractive index unit), and the temperature sensitivity is $51.5 \mathrm{pm} /{ }^{\circ} \mathrm{C}$ at $200 \sim 875^{\circ} \mathrm{C}$ with a step of $25^{\circ} \mathrm{C}$.

\section{Fiber Sensor Fabrication}

The scheme of the fiber sensor fabrication system using a fs laser is shown in Figure 1. The central wavelength, pulse width and repetition rate of the fs laser (Spectra-Physics, Inc.) are $800 \mathrm{~nm}, 35 \mathrm{fs}$ and $1 \mathrm{kHz}$, respectively.

Figure 1. The scheme of the fs laser fabrication system.

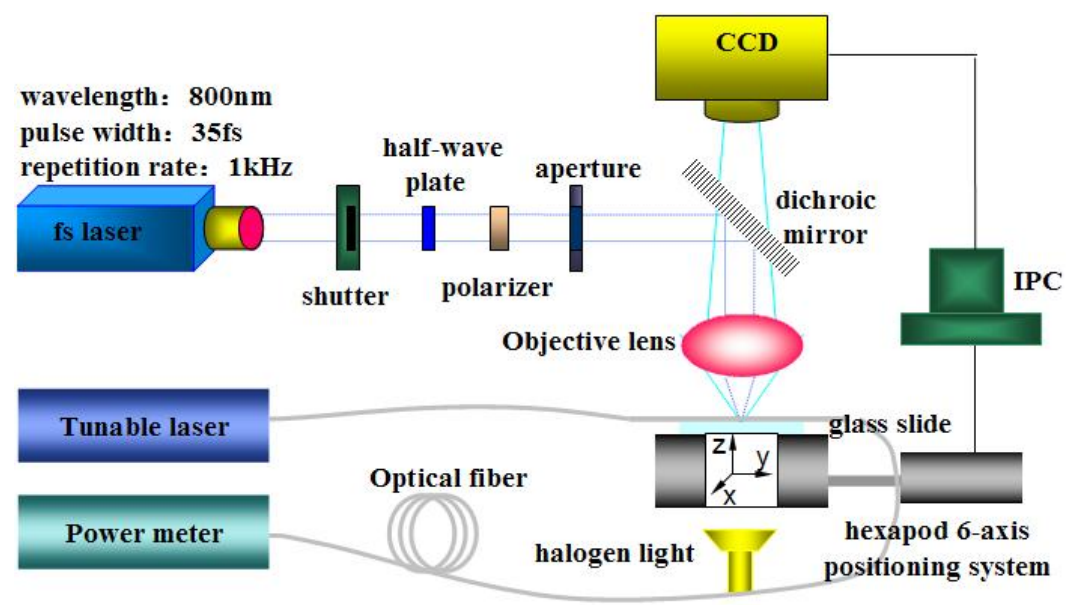


The laser pulse energy is attenuated through a half-wave plate and a polarizer to less than $50 \mu \mathrm{J}$. Then, several neutral density filters are applied to reduce the pulse energy to less than $600 \mathrm{~nJ}$ before the objective lens. The attenuated fs laser beam is focused by an NA $=0.45$ objective lens. The diameters of the single-mode fiber core and cladding are $8.2 \mu \mathrm{m}$ and $125 \mu \mathrm{m}$ respectively. The effective refractive index of the fundamental mode at $1,550 \mathrm{~nm}$ is 1.4682 , and the calculated physical refractive indices of the fiber core and the cladding are about 1.4712 and 1.4659 respectively. A detection system (Agilent 8163B) consisting of a tunable laser and an optical power meter is employed to monitor the transmission spectra by wavelength sweeping. Figure 2 shows the side-ablated structure (an in-line trench) on the single-mode fiber with a length of about $75 \mu \mathrm{m}$. During by fs laser processing of fibers, nitrogen gas is used to blow off debris.

Figure 2. Trench fabricated by fs laser pulses. (a) Structural illustration. (b) Side view (a half part). (c) Cross section.
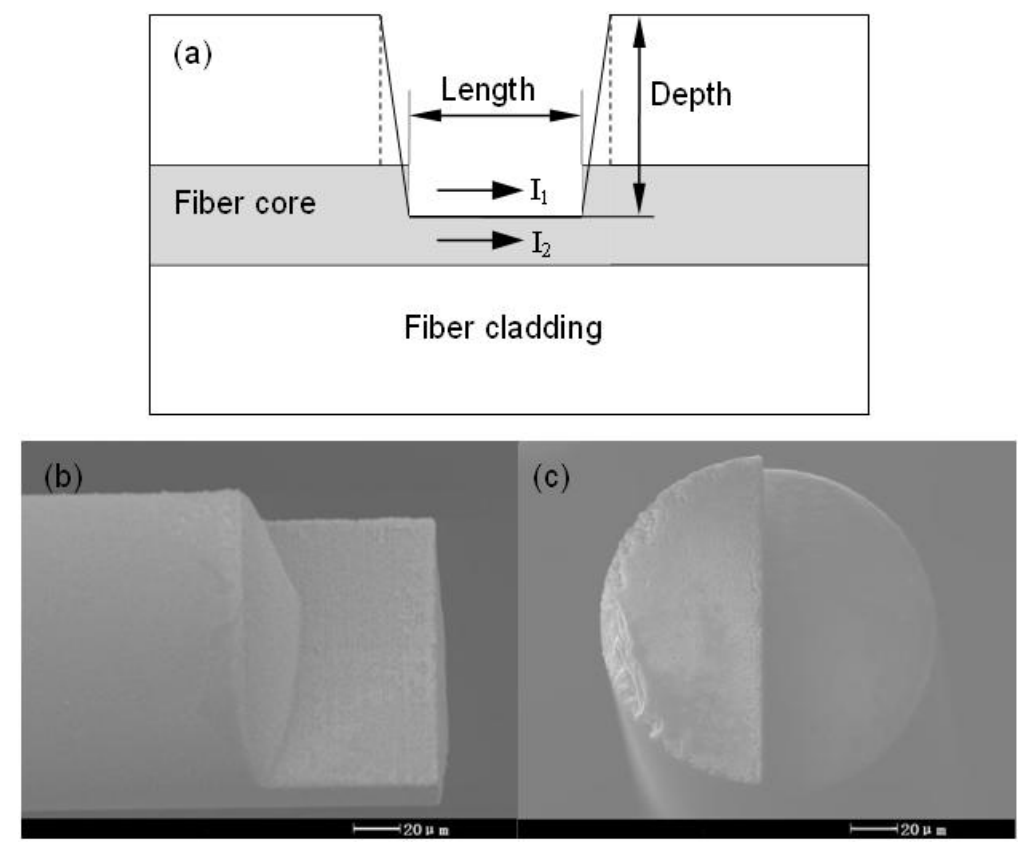

The transmission spectra of the side-ablated fiber were real-time tested during the fs laser fabrication process. The tunable laser continuously scans through its wavelength range $(1,465-1,575 \mathrm{~nm})$ at the rate of $0.5 \mathrm{~nm}$ per step. In each scanning cycle of fs laser ablation, a layer in $\mathrm{x}-\mathrm{y}$ plane (as shown in Figure 1) is exposed to laser irradiation. Transmission spectra evolutions of structures from the $1^{\text {st }}$ scanning cycle to the $8^{\text {th }}$ scanning cycle are shown in Figure 3. The transmission spectra of the processed fiber keep changing in each processing cycle by fs laser ablation. In the $1^{\text {st }}$ scanning cycle, the ablation depth is about $60 \mu \mathrm{m}$, which results in an attenuation band with a relatively low loss. The processing cycle is repeated eight times at the same depth. The losses of the attenuation bands increase from the $1^{\text {st }}$ scanning cycle to the maximum at the $5^{\text {th }}$ scanning cycle and then decrease from the $6^{\text {th }}$ to the $8^{\text {th }}$ cycle. The interference dip wavelength shifts during the ablation processes while FSR nearly keeps constant. From the $1^{\text {st }}$ cycle to the $8^{\text {th }}$ cycle, debris decreases gradually. Fifty fabricated samples demonstrate high repeatability of the trench structure and its sensing properties. 
Figure 3. Transmission spectra evolution of the sensor structure during the $1^{\text {st }}-8^{\text {th }}$ scanning cycle.

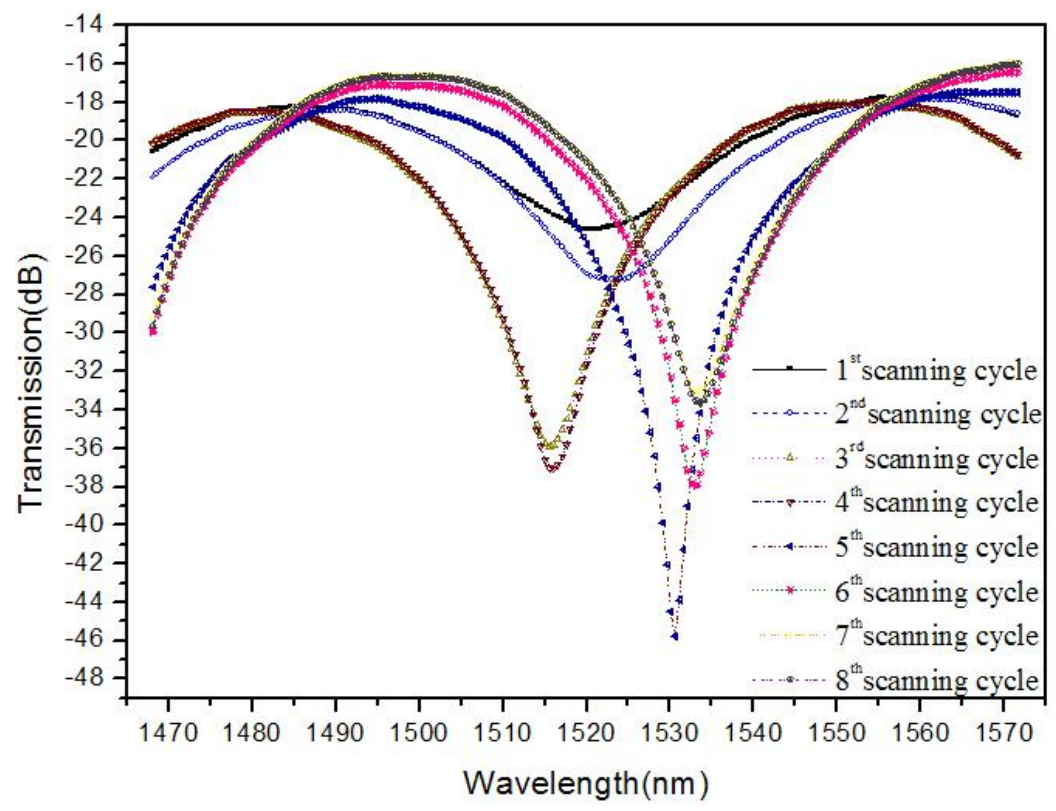

\section{Results and Discussion}

Fifty MZI fiber sensors are fabricated with the same trench depth of $60 \mu \mathrm{m}$ but different lengths of 50, 65, 80, 100 and $115 \mu \mathrm{m}$. As shown in Figure 4, the fringe visibilities of the processed fibers are all greater than $25 \mathrm{~dB}$. The background loss increases as the trench length increases and it is greater than $11 \mathrm{~dB}$ in all the cases, which is similar to the previously reported experiment [1]. The relatively high loss may be mainly due to the light scattering at the laser-ablated surface [11]. At the trench lengths of 50, 65, 80, 100 and $115 \mu \mathrm{m}$, the FSRs are about 101, 74, 64, 53.5 and $38 \mathrm{~nm}$, respectively. This indicates that FSR decreases as the trench length increases, which implies more interference orders at longer trench lengths.

Figure 4. Transmission spectra of the structures at different lengths.

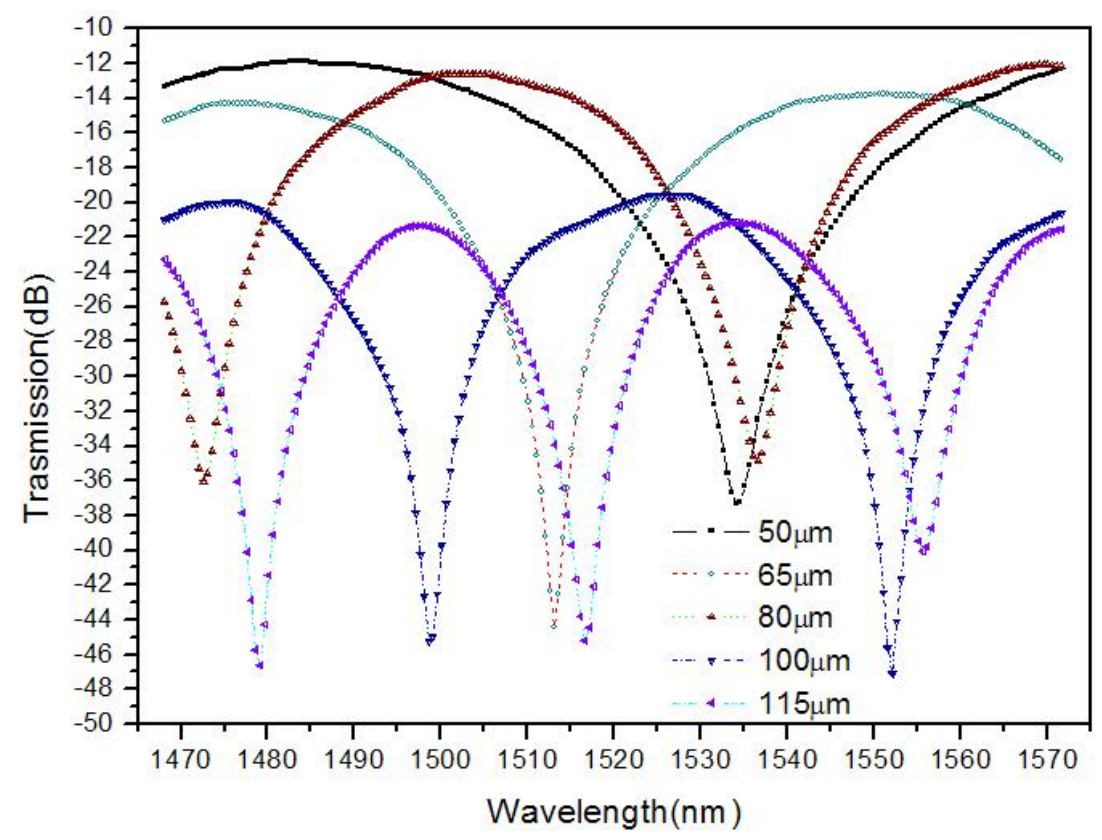


The fabricated structure forms a MZI whose two main light transmission paths are (1) the remaining D-type fiber core; and (2) the cavity in the trench. The interference intensity is expressed by [17]:

$$
I=I_{1}+I_{2}+2 \sqrt{I_{1} I_{2}} \cos \phi
$$

where $I_{1}$ and $I_{2}$ are the intensities along the two light paths and $\phi\left(=2 \pi \Delta n_{\text {eff }} L / \lambda+\varphi_{0}\right)$ is the phase difference; $\Delta n_{\text {eff }}(\approx 0.4682)$ is the difference between effective refractive index of the D-type fiber core and that of the trench cavity; $\lambda$ is the wavelength; $L$ is the trench length; and $\varphi_{0}$ is the initial interference phase. The fringe visibility depends on $I_{1}$ and $I_{2}$, and is optimized when $I_{1}=I_{2}$. The interference changes in each ablation scanning cycle are shown in Figure 3.

According to Equation (1), the phase difference of two adjacent minimum interference signals is $2 \pi$. Therefore:

$$
\left(2 \pi \Delta n_{e f f} L / \lambda_{1}+\varphi_{0}\right)-\left(2 \pi \Delta n_{e f f} L / \lambda_{2}+\varphi_{0}\right)=2 \pi
$$

where $\lambda_{1}$ and $\lambda_{2}$ are the wavelengths corresponding to the two adjacent minimum interference signals.

Thus, the trench length is:

$$
L=\lambda_{1} \lambda_{2} /\left(\Delta n_{e f f}\left(\lambda_{2}-\lambda_{1}\right)\right)
$$

which shows that the FSR decreases as the trench length increases. Based on the interference spectra in Figure 4, the calculated trench lengths are 46.5, 62.8, 75.5, 92.8 and $126.1 \mu \mathrm{m}$, which are reasonably close to the experimental results: $50,65,80,100$ and $115 \mu \mathrm{m}$, respectively. The errors may mainly be caused by the simplification that the cladding effects and variation of $\Delta n_{\text {eff }}$ are not considered.

Gas sensing tests in air and acetone vapor were conducted. The sensor with a trench length of about $80 \mu \mathrm{m}$ was put into a sealed stainless steel tube. The inner diameter and the length of the stainless steel are about $1 \mathrm{~cm}$ and $20 \mathrm{~cm}$, respectively. The sensor transmission spectrum in air at room temperature is shown in Figure 5.

Figure 5. Sensing test results in air and acetone vapor at room temperatures.

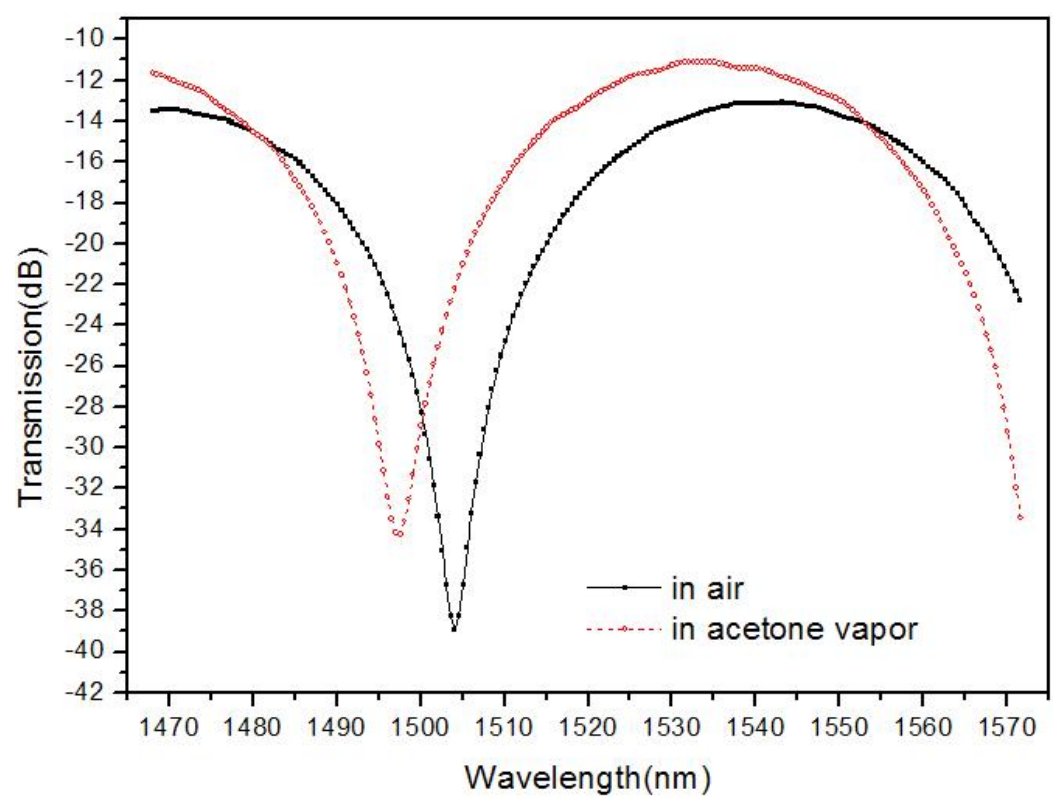


Then, $1.5 \mathrm{~mL}$ acetone was injected into the stainless tube. The transmission spectrum of the sensor in the acetone vapor was measured at room temperature. The spectrum scanning procedure is repeated several times until there is no obvious change compared with the preceding ones and the final sensor spectrum in acetone vapor is also shown in Figure 5. The refractive index of acetone vapor is greater than that of air, between which the difference is on the order of magnitude of $10^{-4}$ RIU. Compared with the results in air, the interference dip wavelength shift in acetone vapor is about $6.5 \mathrm{~nm}$. The sensitivity is about $10^{4} \mathrm{~nm} / \mathrm{RIU}$ for acetone vapor.

Temperature measurements were also conducted by using the proposed fiber sensor. The sensor with a trench length of about $85 \mu \mathrm{m}$ was selected. The temperature changes from $200{ }^{\circ} \mathrm{C}$ to $875{ }^{\circ} \mathrm{C}$ at a step of $25{ }^{\circ} \mathrm{C}$. The interference dip wavelength shows a red shift with the increase of the temperature, as shown in Figure 6. It is mainly due to the change of $\Delta n_{\text {eff }}$ or the effective refractive index change of the D-type fiber caused by the temperature variation. The temperature sensitivity estimated by least square linear fitting is $51.5 \mathrm{pm} /{ }^{\circ} \mathrm{C}$.

Figure 6. The temperature sensing property of the sensor.

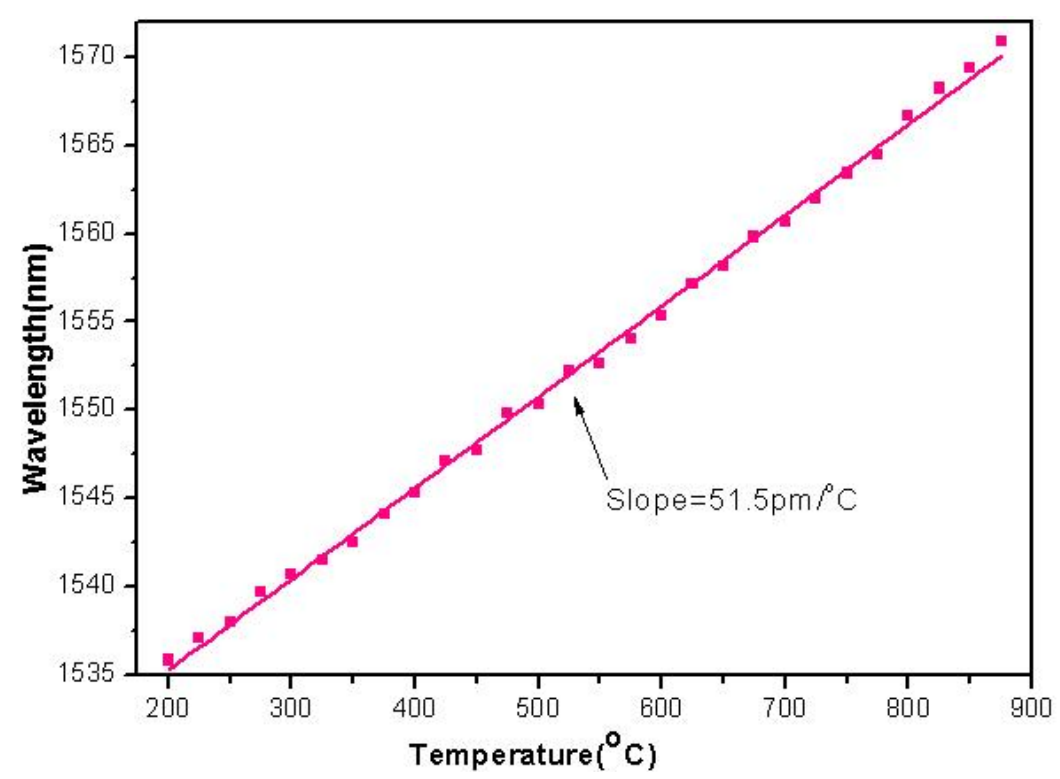

\section{Conclusions}

This paper reports a MZI sensor ablated by fs laser pulses in single-mode fibers, which demonstrates exciting high-quality for both temperature and chemical sensing. For 50 processed samples, the sensing structure showed very high fabrication and sensing repeatability, and structures with the desired proprieties are successfully obtained in every experiment. The sensing mechanism is discussed theoretically, and is in agreement with the experimental results. The fringe visibility of the structure is more than $25 \mathrm{~dB}$. The sensitivity for acetone vapor sensing is about $10^{4} \mathrm{~nm} / \mathrm{RIU}$. The sensor was applied for high temperature measurements with a sensitivity of $51.5 \mathrm{pm} /{ }^{\circ} \mathrm{C}$ at $200 \sim 875^{\circ} \mathrm{C}$. 


\section{Acknowledgements}

This research is supported by the National Natural Science Foundation of China (Grant No. 90923039 and 50705009) and 863 of Ministry of Science and Technology of China (Grant No. 2008AA03Z301).

\section{References}

1. Andreev, A.T.; Zafirova, B.S.; Karakoleva, E.I. Single-mode fiber polished into the core as a sensor element. Sens. Actuat. A-Phys. 1998, 64, 209-212.

2. Sohn, K.R.; Kim, K.T.; Song, J.W. Optical fiber sensor for water detection using a side-polished fiber coupler with a planar glass-overlay-waveguide. Sens. Actuat. A-Phys. 2002, 101, 137-142.

3. Tan, W.; Lei, S.; Chen, X. Modeling of an optical sensor based on Whispering Gallery Modes (WGMs) on the surface guiding layer of glass filaments. Sensors 2008, 8, 6761-6768.

4. Sun, H.; Pyajt, A.; Luo, J.; Shi, Z.; Hau, S.; Jen, A.K.Y.; Dalton, L.R.; Chen, A. All-dielectric electrooptic sensor based on a polymer microresonator coupled side-polished optical fiber. IEEE Sens. J. 2007, 7, 515-524.

5. McCallion, K.; Johnstone, W.; Fawcett, G. Tunable in-line fiber-optic bandpass filter. Opt. Lett. 1994, 19, 542-544.

6. McCallion, K.; Creaney, S.; Madden, I.; Johnstone, W. A tunable fiber-optic bandpass filter based on polished fiber to planar waveguide coupling techniques. Opt. Fiber Technol. 1995, 1, 271-277.

7. Chen, N.K.; Chi, S.; Tseng, S.M. Wideband tunable fiber short-pass filter based on side-polished fiberwith dispersive polymer overlay. Opt. Lett. 2004, 29, 2219-2221.

8. Tseng, S.M.; Chen, C.L. Side-polished fibers. Appl. Opt. 1992, 31, 3438-3447.

9. Rao, Y.J.; Deng, M.; Duan, D.W.; Yang, X.C.; Zhu, T.; Cheng, G.H. Micro fabry-perot interferometers in silica fibers machined by femtosecond laser. Opt. Express 2007, 15, 14123-14128.

10. Chen, C.H.; Chao, T.C.; Li, W.Y.; Shen, W.C.; Cheng, C.W.; Tang, J.L.; Chau, L.K.; Wu, W.T. Novel D-type fiber optic localized plasmon resonance sensor realized by femtosecond laser engraving. J. Laser Micro/Nano Eng. 2010, 5, 1-5.

11. Wei, T.; Han, Y.; Tsai, H.L.; Xiao, H. Miniaturized fiber inline Fabry-Perot interferometer fabricated with a femtosecond laser. Opt. Lett. 2008, 33, 536-538.

12. Jiang, L.; Tsai, H.L. Repeatable nanostructures in dielectrics by femtosecond laser pulse trains. Appl. Phys. Lett. 2005, 87, 151104-151106.

13. Lin, C.H.; Jiang, L.; Zhou, J.; Xiao, H.; Chen, S.J.; Tsai, H.L. Laser-treated substrate with nanoparticles for surface-enhanced Raman scattering. Opt. Lett. 2010, 35, 941-943.

14. Wei, T.; Han, Y.; Li, Y.; Tsai, H.L.; Xiao, H. Temperature-insensitive miniaturized fiber inline Fabry-Perot interferometer for highly sensitive refractive index measurement. Opt. Express 2008, 16, 5764-5769.

15. Chen, C.H.; Tsao, T.C.; Li, W.Y.; Shen, W.C.; Cheng, C.W.; Tang, J.L.; Jen, C.P.; Chau, L.K.; $\mathrm{Wu}, \mathrm{W} . \mathrm{T}$. Novel U-shape gold nanoparticles-modified optical fiber for localized plasmon resonance chemical sensing. Microsyst. Technol. 2010, 16, 1207-1214. 
16. Chen, C.H.; Tsao, T.C.; Tang, J.L.; Wu, W.T. A multi-D-shaped optical fiber for refractive index sensing. Sensors 2010, 10, 4794-4804.

17. Jung, Y.; Lee, S.; Lee, B.H.; Oh, K. Ultracompact in-line broadband Mach-Zehnder interferometer using a composite leaky hollow-optical-fiber waveguide. Opt. Lett. 2008, 33, 2934-2936.

(C) 2010 by the authors; licensee MDPI, Basel, Switzerland. This article is an open access article distributed under the terms and conditions of the Creative Commons Attribution license (http://creativecommons.org/licenses/by/3.0/). 